\title{
Airway compression management in late-presenting absent pulmonary valve syndrome
}

\author{
Anastasia Martinez-Esteve Melnikova, ${ }^{1}$ Tornike Sologashvili ${ }^{2}$ Maurice Beghetti, ${ }^{1}$ Cécile Tissot, ${ }^{1}$ \\ Afksendiyos Kalangos, ${ }^{2}$ Regula Corbelli, ${ }^{3}$ Yacine Aggoun, ${ }^{1}$ Dominique Didier, ${ }^{4}$ Patrick O. Myers ${ }^{2}$ \\ ${ }^{1}$ Pediatric Cardiology Unit, Children's University Hospital; ${ }^{2}$ Division of Cardiovascular Surgery, Faculty of Medicine, \\ Geneva University Hospitals; ${ }^{3}$ Unit of Pediatric Pneumology, Children's University Hospital; ${ }^{4}$ Department of Radiology, \\ Faculty of Medicine, Geneva University Hospitals, Geneva, Switzerland
}

\begin{abstract}
Introduction: Patients with absent pulmonary valve syndrome often present early with airway compression from aneurysmal pulmonary arteries. This study reviews our experience in managing absent pulmonary valve syndrome in later presenting children, and techniques used for managing airway compression. Methods: This study is a retrospective chart review of all patients who underwent repair of absent pulmonary valve syndrome from 2000 to 2012 at our institution. The primary endpoints were post-operative bronchoscopic and clinical evidence of persistent airway compression and need for reinterventions on the pulmonary arteries. Results: A total of 19 patients were included during the study period. The mean age at repair was $4.1 \pm 3.0$ years (range 10 months -11 years). In all, seven patients had pre-operative bronchoscopic evidence of airway compression, which was managed by pulmonary artery reduction plasty in four patients and Lecompte manoeuvre in three patients. There were no peri-operative deaths. In patients with pulmonary artery plasty, two had no post-operative airway compression, one patient had improved compression, and one patient had unchanged compression. In patients managed with a Lecompte manoeuvre, two patients had no or trivial airway compression and one had improved compression. There were six late reinterventions or reoperations on the pulmonary arteries - two out of four in the pulmonary artery plasty group and one out of three in the Lecompte group. Conclusions: Most late-presenting patients with absent pulmonary valve syndrome do not have airway compression. Either pulmonary artery reduction plasty or the Lecompte manoeuvre can relieve proximal airway compression, without a significantly different risk of pulmonary artery reintervention between techniques.
\end{abstract}

Keywords: Absent pulmonary valve syndrome; airway compression; surgical repair; bronchoscopy

Received: 6 August 2013; Accepted: 31 October 2013; First published online: 2 January 2014

A BSENT PULMONARY VALVE SYNDROME IS A RARE conotruncal variant, occurring in $3-6 \%$ of patients with tetralogy of Fallot. It is characterised by a ventricular septal defect that is usually located in the outlet septum, absent or rudimentary pulmonary valve

Correspondence to: Dr P. O. Myers, MD, Division of Cardiovascular Surgery, Faculty of Medicine, Geneva University Hospitals \& School of Medicine, 4 rue Gabrielle-Perret-Gentil 1211 Geneva 14, Switzerland. Tel: + 41 (22) 372-7638; Fax: + 41 (22) 372-7634; E-mail: patrick.myers@hcuge.ch

Presented at the AEPC 47th annual meeting, 22-25 May 2013, London, United Kingdom. leaflets with obstruction at the pulmonary valve ring level, which is densely fibrotic, and an absence of the arterial duct. ${ }^{1}$ The pulmonary arteries are massively dilated and have abnormal branching patterns, often causing severe tracheobronchial obstruction ${ }^{2,3}$ (see Fig 1). The timing of presentation often reflects the degree of airway compression, and appears bimodal, with severe symptoms in some neonates and young infants, and later presentation in older children.

Surgical repair of absent pulmonary valve syndrome usually includes pulmonary artery reduction 


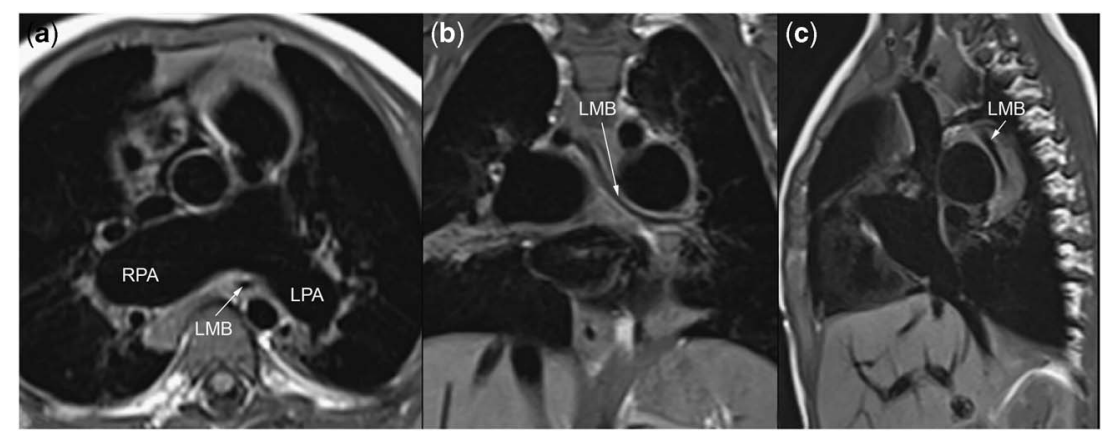

Figure 1.

Cardiac magnetic resonance imaging of bronchial stenosis in absent pulmonary valve syndrome. (a) axial plane, (b) coronal plane, (c) sagittal plane. $L M B=$ left mainstem bronchus; $L P A=$ left pulmonary artery; $R P A=$ right pulmonary artery.

plasty to relieve proximal obstruction of the mainstem airways. ${ }^{1,4,5}$ The Lecompte manoeuvre has been proposed to address airway obstruction, although there are limited data available. ${ }^{6-8}$ There has been no bronchoscopic evaluation of improvement in airway obstruction following repair of absent pulmonary valve syndrome associated with different surgical techniques aimed at relieving obstruction by aneurysmal pulmonary arteries. This study reviews our recent experience in managing absent pulmonary valve syndrome in later presenting children, and surgical techniques used for managing airway compression.

\section{Materials and methods}

\section{Study design}

This study is a retrospective review of all patients who underwent surgical repair of tetralogy of Fallot and absent pulmonary valve syndrome from 2000 to 2012 at our institution. The study was approved by the Institutional Internal Review Board, and individual patient consent was waived.

The primary endpoints were post-operative bronchoscopic and clinical evidence of persistent airway compression, and need for reinterventions or reoperations on the pulmonary arteries. All patients were followed-up to death or until January, 2013. A portion of the patients included were reported previously in brief reports. ${ }^{8,9}$

\section{Surgical technique}

All patients who had pre-operative respiratory symptoms underwent bronchoscopy before the initiation of cardiopulmonary bypass, and after weaning from cardiopulmonary bypass, to assess airway compression by the pulmonary arteries. Imaging of the airways, either through computed tomography or magnetic resonance imaging, are not part of our routine protocol for airway assessment in these patients, and was used to assess associated anomalies as clinically indicated.
All operations were performed through a median sternotomy with normothermic cardiopulmonary bypass, established by aortic and bicaval cannulation. The aortic cannulation and cross-clamp were placed in the distal ascending aorta to allow subsequent aortic transection, if necessary. Myocardial protection consisted of hyperkalaemic crystalloid solution administered into the aortic root. The atrial septal defect was closed primarily through a right atriotomy. A vertical infundibulotomy was then performed, hypertrophied right ventricular muscle bundles were resected, and the ventricular septal defect was closed with a pericardial patch.

In patients with pre-operative bronchoscopic evidence of airway compression, the left and right pulmonary arteries and the first hilar branches were dissected free and mobilised, and airway compression was managed either by incising and reducing the branch pulmonary arteries from hilum to hilum (see Fig 2) or by transecting the aorta and resecting a triangular segment of the aorta, then bringing the pulmonary arteries anterior to the aorta in a Lecompte manoeuvre before completing the aortic anastomosis (see Fig 3). The management choice between Lecompte or pulmonary artery plasty was at the operating surgeon's discretion. The right ventricular outflow tract was reconstructed with a valved conduit.

\section{Statistical analysis}

Statistical analyses were performed with SPSS software (version 21, SPSS Inc., Chicago, Illinois, United States of America). Data are presented as mean \pm standard deviation or median (range) where appropriate. Continuous variables were analysed with the Student's t-test for normally distributed variables, or the related samples Wilcoxon's signed rank test for non-normally distributed variables, and categorical variables using Fisher's exact test. Normality of continuous data was evaluated with the Kolmogorov-Smirnov test. 
(a)

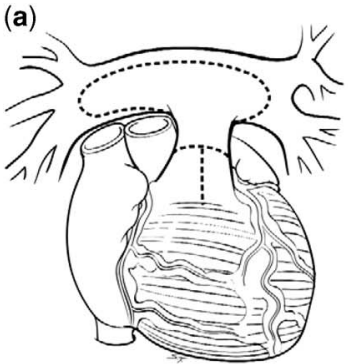

(b)

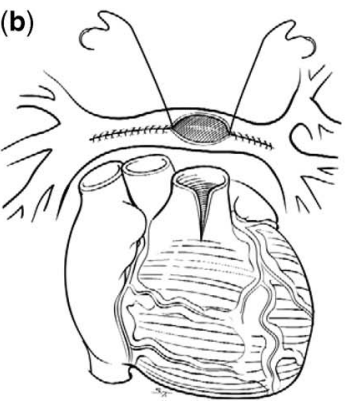

(c)

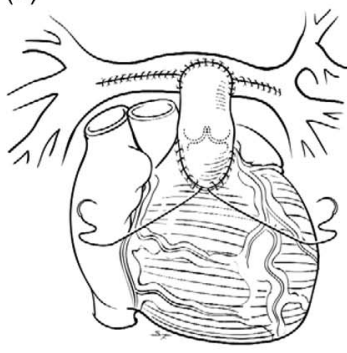

Figure 2.

Pulmonary artery reduction plasty. The pulmonary artery is transected at the annulus, extending across the fibrous ring into the right ventricular infundibulum. Arterioplasty of the branch pulmonary arteries reduces their calibre, and a valved conduit is placed between the right ventriculotomy and the pulmonary bifurcation. Reproduced with permission from Brown et al. ${ }^{2}$

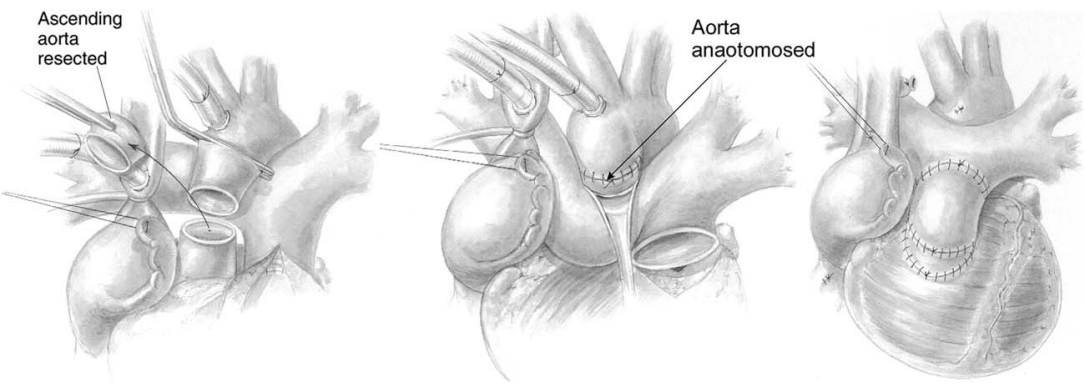

Figure 3.

Lecompte manoeuvre in tetralogy of Fallot with absent pulmonary valve syndrome. The aorta is transected above at the sinotubular junction, and a short tubular section of the aorta is resected, to bring the ascending aorta down and to the left. The pulmonary artery is transected above the fibrous annulus, avoiding the left coronary artery, and passed anterior to the aorta, after mobilisation of the right pulmonary artery to avoid compression of the right coronary. Reproduced with permission from Hraska. ${ }^{3}$

Table 1. Patient baseline characteristics.

\begin{tabular}{lc}
\hline Characteristic & Value \\
\hline Patients & 19 \\
Age at repair (years) & $4.1 \pm 3.0$ \\
$\quad$ Infants & $1(5.2 \%)$ \\
Associated anomalies & $2(10.5 \%)$ \\
$\quad$ Discontinuous left pulmonary artery from major aortopulmonary collaterals & $1(5.2 \%)$ \\
Major aortopulmonary collateral to the left pulmonary artery & $1(5.2 \%)$ \\
Left pulmonary artery hypoplasia & $1(5.2 \%)$ \\
Right aortic arch & $1(5.2 \%)$ \\
Supracardiac totally anomalous pulmonary venous return, infradiaphragmatic major aortopulmonary collateral to the right lung & \\
Arterial duct & $1(5.2 \%)$ \\
DiGeorge syndrome & $1(5.2 \%)$ \\
Pre-operative respiratory symptoms & $8(42.1 \%)$ \\
Pre-operative bronchoscopic airway compression & $7(36.8 \%)$ \\
\hline
\end{tabular}

\section{Results}

\section{Demographics}

A total of 19 patients were included during the study period. Patient baseline characteristics are summarised in Table 1 . The mean age at repair was $4.1 \pm 3.0$ years (range 10 months-11 years). As a tertiary referral centre for humanitarian patients, only one 10-month-old infant was included during the study period.

In all, six patients had associated anomalies: three with discontinuous left pulmonary artery from major aortopulmonary collaterals, one with a major aortopulmonary collateral to the left pulmonary artery, one with a right aortic arch, supracardiac totally 
Table 2. Operative techniques.

\begin{tabular}{lc}
\hline Characteristic & Value \\
\hline Right ventricular-to-pulmonary artery conduit & $19(100 \%)$ \\
Matrix P plus & $4(21.1 \%)$ \\
Contegra & $8(42.1 \%)$ \\
Homograft & $6(31.6 \%)$ \\
Labcor & $1(5.3 \%)$ \\
Conduit size & $15.7 \pm 1.8 \mathrm{~mm}$ \\
Associated procedures & \\
Pulmonary artery reduction plasty & $4(21.1 \%)$ \\
Lecompte manoeuvre & $3(15.8 \%)$ \\
Branch pulmonary artery patch augmentation & $1(5.2 \%)$ \\
Pulmonary artery collateral unifocalisation & $2(10.5 \%)$ \\
Arterial duct ligation & $1(5.2 \%)$ \\
\hline
\end{tabular}

anomalous pulmonary venous return, and infradiaphragmatic major aortopulmonary collateral to the right lung, and one patient with DiGeorge syndrome. In all, eight patients with respiratory symptoms had pre-operative bronchoscopy, which showed airway compression in seven patients.

\section{Operative details and outcomes}

Surgical management is detailed in Table 2. Airway compression was addressed in all seven patients with pre-operative bronchoscopic evidence of compression, by pulmonary artery reduction plasty in four patients, and Lecompte manoeuvre in three patients.

There were no peri-operative deaths. In all, three patients presented complete heart block, requiring permanent pacemaker implantation.

\section{Post-operative airway compression}

Among the four patients managed with pulmonary artery reduction plasty, two patients had no residual airway compression, one patient had improved compression, and one patient had unchanged compression on post-operative bronchoscopy. In patients managed with a Lecompte manoeuvre, two patients had no or trivial airway compression and one had improved compression. All of the patients were discharged without respiratory symptoms.

\section{Follow-up}

During a median follow-up of 6.2 years (range 14 months-13 years), there were no late deaths. None of the patients presented recurrent respiratory symptoms. There were six late reinterventions or reoperations on the pulmonary arteries. There were two patients who required surgical replacement of their right ventricle to pulmonary artery conduit 1 contegra, 1 homograft - one patient who required balloon dilatation of the distal conduit anastomosis, two patients who required right pulmonary artery balloon dilatation and stenting, and one patient who had a percutaneous pulmonary valve replacement with a Melody ${ }^{\circledR}$ valve. The distribution of reinterventions and reoperations was not significantly different if and how airway compression was managed at repair: two out of four in the pulmonary artery plasty group and one out of three in the Lecompte group.

Finally, two patients have significant residual haemodynamic lesions that are being treated conservatively: one patient presented moderate stenosis at the right ventricular-pulmonary artery conduit anastomosis (Labcor), which is being managed conservatively and one patient presented moderate stenosis at the left pulmonary artery unifocalisation to a Gore-Tex tube graft, which has not been reintervened on.

\section{Discussion}

Tetralogy of Fallot with absent pulmonary valve syndrome is a rare congenital cardiac malformation, comprising 3-6\% of patients of the tetralogy complex. ${ }^{3}$ Chevers first described this syndrome in $1847,{ }^{10}$ as the association of a malalignment ventricular septal defect and stenosis at the pulmonary artery annulus with or without narrowing of the right ventricular infundibulum, and two additional unique features, pulmonary regurgitation secondary to absent or rudimentary pulmonary valve leaflets and aneurysmal dilatation of the pulmonary trunk and the main pulmonary arterial branches. It is strongly associated with DiGeorge syndrome, present in up to $25 \%$ of patients with absent pulmonary valve syndrome. ${ }^{11}$

Clinical presentation reflects the degree of respiratory distress secondary to airway obstruction and infections and heart failure as a result of the leftto-right shunt. ${ }^{3}$ There is a bimodal presentation. The first group comprises neonates and infants with severe respiratory distress, and the mortality in this patient group remains considerable. ${ }^{2,3}$ Massively dilated right and left pulmonary arteries up to the hilum are amenable to surgical intervention. However, abnormalities of arborisation and intrapulmonary bronchial compression cannot be addressed during surgery. This could partially explain the high rate of failure with the treatment of the youngest, symptomatic group of patients. Repair in older patients is deemed lower risk, although there is a lack of recent data in this patient group. We report our experience in managing patients with absent pulmonary valve syndrome mostly at a relatively older age, from toddlerhood to 10 years of age. Our patients had more associated anomalies, such as non-confluent pulmonary arteries and major aortopulmonary collaterals, which may explain the relatively high rate of reoperation and reintervention on the conduits 
and branch pulmonary arteries. Furthermore, our institutional approach is to use valve conduits to re-establish the right ventricular-to-pulmonary artery continuity, associated with a significant burden of conduit stenosis with time calcification. Although many authors have advocated using valved conduits in neonates because of the risk of pulmonary hypertension, most favour using transannular patch to delay reoperations as long as possible.

Significant controversy persists regarding the management of patients with absent pulmonary valve syndrome, and particularly how to manage airway obstruction. A number of surgical techniques for reduction of bronchial obstruction have been proposed, with variable results. Palliative operations, as summarised by Brown et al, ${ }^{2}$ included pulmonary artery or aortopexy to the chest wall, transecting the right pulmonary artery and reattaching it to the pulmonary trunk in front of the aorta (pseudoLecompte), creation of a classic Glenn shunt, reducing the pulmonary artery size, lobectomies of collapsed or emphysematous lungs, and pulmonary artery ligation, and construction of a central shunt. Most recent reports have focused on reducing the size of the pulmonary arteries, by resecting a segment of anterior pulmonary and branch pulmonary arterial wall and closing the defect, usually over an appropriately sized dilator, and closing the main pulmonary artery with a transannular patch or a pulmonary valve replacement, and adding posterior plication in severely dilated pulmonary arteries. ${ }^{1,2,4,5,12}$ The Lecompte manoeuvre has been proposed as an alternate solution to airway compression, particularly in neonates or young infants, as a means to pull the dilated pulmonary arteries away from the airway and relieve obstruction. Hraska et $\mathrm{al}^{6}$ first reported their intermediate results in six young infants using this technique, with post-operative computed tomographic scans showing no proximal airway compression. Nölke et al reported their experience in four patients - three infants with tetralogy of Fallot, absent pulmonary valve syndrome, and one 3.5-yearold child with an intact ventricular septum - who underwent repair including the Lecompte manoeuvre. They reported no deaths and relief of airway obstruction in all patients at a mean follow-up of 2 years, with the exception of one patient with pre-operative subglottic stenosis requiring tracheostomy. The patients we report herein are relatively older, and addressing airway compression at this late stage could risk unmasking tracheo-bronchomalacia. This complication has not been evident in our experience, although this may be due to the fact that our patients had relatively mild respiratory symptoms and presented later for repair. Given the limited number of patients included and the power of this study, these data are not able to show superiority of either surgical techniques for relieving airway obstruction, and we have individualised the surgical technique to individual patient anatomy. If the branch pulmonary arteries are very large and mechanically compress the airways because of their shear size, we prefer to use reduction arterioplasty. If there is evidence of airway obstruction with relatively normal-sized or slightly enlarged branch pulmonary arteries, we suspect a component of bronchomalacia or distal airway compression, and prefer to use the Lecompte manoeuvre to pull the pulmonary arteries away from the airways.

From a technical perspective, Hraska noted two surgical pitfalls of this technique ${ }^{3}$ : the azygos vein is systematically ligated and divided, to improve mobilisation of the right pulmonary artery; and the right coronary artery is potentially at risk of compression by the right pulmonary artery if it is insufficiently mobilised and "bow-stringed" to achieve a direct connection of the pulmonary artery to the right ventricular infundibulum. As we routinely use valved conduits to reconstruct the right ventricularpulmonary artery continuity and possibly because of our relatively older patient population, we have not found it necessary to divide the azygos vein or encountered right coronary artery compression.

There is significant controversy regarding the use of valved conduits for patients with absent pulmonary valve. Placing a conduit has the advantage of avoiding the volume load on an already dilated right ventricle after repair, and is particularly indicated in patients with high pulmonary arterial pressure. However, patients with absent pulmonary valve syndrome have a relatively protected pulmonary vascular bed because of the obstruction at the level of the pulmonary annular ring, and rarely present pulmonary hypertension. Furthermore, placing a valved conduit that will calcify and become stenotic within a few years, with the added risk of stenosis at the distal anastomosis, runs the risk of more frequent reoperations for conduit replacement compared with a transannular patch. Interestingly, two of our patients required branch pulmonary artery balloon dilatation and stenting, which is somewhat surprising in the setting of aneurysmal pulmonary arteries. Of these patients, one had discontinuous pulmonary arteries, with a left pulmonary artery arising from a major aortopulmonary collateral, which had to be unifocalised at the time of absent pulmonary valve syndrome repair. This patient subsequently presented with distal conduit and proximal left pulmonary artery stenosis, which required balloon dilatation and stenting. The other patient, who underwent Lecompte manoeuvre for airway compression management, presented stenosis of the distal anastomosis of his Contegra conduit, which encroached on the ostium 
of both branch pulmonary arteries. This required balloon dilatation and stenting of the proximal branch pulmonary arteries. A transannular patch places a volume load on the right ventricle, which will later require pulmonary valve replacement, although this will usually occur later than with a valved conduit. Unfortunately, there are no data within this patient population to guide which is the better approach, although most will agree that a symptomatic neonate with absent pulmonary valve syndrome will be far better served with a valved conduit. We favour placing a valved conduit, as this allows for less inotropic support and faster time to extubation in the intensive care unit, and as several of our patients had pulmonary arterial lesions that could lead to high post-operative pulmonary arterial pressure - discontinuous pulmonary arteries and/or hypoplastic branch pulmonary arteries.

This study has several limitations. It is a retrospective review of management of absent pulmonary valve syndrome, and represents a 13-year period of evolving experience with managing this disease, from a single institution. As such, any inferences drawn from these data are limited by confounding variables, such as repair type, associated anomalies, and peri-operative management. Our sample size also limits our ability to perform extensive statistical adjustments for these differences. Furthermore, the choice of repair to relieve airway obstruction was at the discretion of the operating surgeon, introducing the risk of selection bias. Finally, airway evaluation by bronchoscopy was performed solely in symptomatic patients. Some asymptomatic patients might have had airway compression or anomalies. Nonetheless, this study reports one of the largest number of patients presenting later with absent pulmonary valve syndrome.

In conclusion, a majority of late-presenting patients with absent pulmonary valve syndrome do not have symptoms or signs of airway compression. When present, either pulmonary artery reduction plasty or the Lecompte manoeuvre can relieve proximal airway compression, without a significantly different risk of pulmonary artery reintervention between techniques.

\section{Acknowledgements}

None.

\section{Financial Support}

This research received no specific grant from any funding agency, commercial or not-for-profit sectors.

\section{Conflicts of Interest}

None.

\section{Ethical Standards}

The authors assert that all procedures contributing to this work comply with the ethical standards of the relevant national guidelines on human experimentation and with the Helsinki Declaration of 1975 , as revised in 2008, and has been approved by the Geneva University Hospitals ethics committee on research on human subjects (HUG-CEREH).

\section{References}

1. Snir E, de Leval MR, Elliott MJ, Stark J. Current surgical technique to repair Fallot's tetralogy with absent pulmonary valve syndrome. Ann Thorac Surg 1991; 51: 979-982.

2. Brown JW, Ruzmetov M, Vijay P, Rodefeld MD, Turrentine MW. Surgical treatment of absent pulmonary valve syndrome associated with bronchial obstruction. Ann Thorac Surg 2006; 82: 2221-2226.

3. Hraska V. Absent pulmonary valve repair. Op Techn Thorac Cardiovasc Surg 2007; 12: 36-46.

4. Chen JM, Glickstein JS, Margossian R, et al. Superior outcomes for repair in infants and neonates with tetralogy of Fallot with absent pulmonary valve syndrome. J Thorac Cardiovasc Surg 2006; 132: 1099-1104.

5. Conte S, Serraf A, Godart F, et al. Technique to repair tetralogy of Fallot with absent pulmonary valve. Ann Thorac Surg 1997; 63: 1489-1491.

6. Hraska V, Kantorova A, Kunovsky P, Haviar D. Intermediate results with correction of tetralogy of Fallot with absent pulmonary valve using a new approach. Eur J Cardiothorac Surg 2002; 21: 711-714; discussion 714-715.

7. Nolke L, Azakie A, Anagnostopoulos PV, Alphonso N, Karl TR. The Lecompte maneuver for relief of airway compression in absent pulmonary valve syndrome. Ann Thorac Surg 2006; 81: 1802-1807.

8. Tissot C, Aggoun Y, Beghetti M, et al. The Lecompte maneuver as an alternative to reduction pulmonary arterioplasty for relief of airway compression in absent pulmonary valve syndrome. Ann Thorac Surg 2007; 83: 727; author reply 727-728.

9. Myers PO, Kalangos A, Didier D, Tissot C. Tetralogy of Fallot with absent pulmonary valve, supracardiac total anomalous pulmonary venous connection, and infradiaphragmatic systemic to pulmonary collateral artery. Eur Heart J 2010; 31: 3005.

10. Chevers N. Recherches sur les maladies de l'artère pulmonaire. Arch Gen Med 1847; 15: 488-508.

11. Volpe P, Paladini D, Marasini M, et al. Characteristics, associations and outcome of absent pulmonary valve syndrome in the fetus. Ultrasound Obstet Gynecol 2004; 24: 623-628.

12. Kirshbom PM, Kogon BE. Tetralogy of Fallot with absent pulmonary valve syndrome. Semin Thorac Cardiovasc Surg Pediatr Card Surg Annu 2004; 7: 65-71. 